\title{
Generalized Particle Statistics in Two-Dimensions: Examples from the Theory of Free Massive Dirac Field
}

\author{
Dario Salvitti \\ Dipartimento di Matematica, Università di Roma "La Sapienza" \\ P.le Aldo Moro 2, 00185 Roma, Italy \\ salvitti@mat.uniroma1.it
}

Dedicated to the memory of Sabrina Picucci

\begin{abstract}
In the framework of algebraic quantum field theory we analyze the anomalous statistics exhibited by a class of automorphisms of the observable algebra of the two-dimensional free massive Dirac field, constructed by fermionic gauge group methods. The violation of Haag duality, the topological peculiarity of a two-dimensional space-time and the fact that unitary implementers do not lie in the global field algebra account for strange behaviour of statistics, which is no longer an intrinsic property of sectors. Since automorphisms are not inner, we exploit asymptotic abelianness of intertwiners in order to construct a braiding for a suitable $C^{*}$-tensor subcategory of $\operatorname{End}(\mathscr{A})$. We define two inequivalent classes of path connected bi-asymptopias, selecting only those sets of nets which yield a true generalized statistics operator.
\end{abstract}

\section{Introduction}

The intrinsic definition of particle statistics in the approach of Algebraic Quantum Field Theory (AQFT) in a four-dimensional space-time is provided by assigning to superselection sectors equivalence classes of permutation group representations, which describe the statistics of multiparticle states. In a $(3+1)$-dimensional space-time, fields and particles obey Bose-Fermi alternative, exhibiting the more general bosonic or fermionic parastatistics, while in lower dimensional Minkowski space statistics are described, in general, by braid group representations. The first models leading to particles described by a one-dimensional representation of the braid group (anyons) are in [25], while higher dimensional representations describe plektons. In a $(2+1)$-dimensional space-time, strictly local quantum fields are always subject to the normal commutation rules, but particles carrying "topological 
charges", created from the vacuum by the action of fields localized in cones, may exhibit intermediate statistics.

The statistics of a sector describes the interchange of identical charges. In two dimensions, DHR theory allows for two distinct statistics operators (one the inverse of the other), since the causal complement of a bounded region has two connected components. The statistics operator is a topological invariant if the pairs of spatially separated auxiliary regions can be continuously deformed from one to the other, maintaining a relative spacelike distance. Therefore, the braid group enters in the description of DHR superselection charges localized in two-dimensional double cones, for intervals of the real line or in $(2+1)$-dimensional theories for charges localized in space-like cones.

Superselection sectors in four-dimensional theories are classified by equivalence classes of irreducible representations of the compact group of internal symmetries [12. However, if the superselection category in low dimensional theories is not symmetric but only braided, such a group may not exist. Indeed, some models of (1+1)-dimensional conformal fields exhibit a superselection structure which seems not to fit any representation group theory.

Why "generalized" particles statistics? Well, this is necessary since the algebraic approach to local field theories which do not fulfill Haag duality and which does not allow non inner automorphisms of the underlying field algebra does not yield a well defined notion of statistics. Thus, we need to extend it to physical theories which do not fit the prescriptions of the algebraic framework totally, as in the case of smeared-out kink operators [20] in the context of the two-dimensional free massive Dirac field in the formalism of relativistic second quantization developed in [5]. There, not only is Haag duality violated (Sect. 3), but a family of unitary operators implementing DHR automorphisms is not in the field algebra, forcing us to explore alternative tools. In a more general setting, field theories in $(1+1)$-dimensions satisfying twisted Haag duality and the split property for wedges and having an unbroken (i.e. unitarily implemented) group of inner symmetries $G$ give rise to a not Haag dual observable algebra $\mathscr{A}=\mathscr{F}^{G}[16$. Split property for wedges has been proven recently by Buchholz and Lechner for the Bose and Fermi cases [3]. Together with the argument in [16], this proves that that the observable algebra is not Haag dual when $\mathscr{F}$ is any finite product of free massive Bose and Fermi fields and $G$ is non-trivial.

We remark that "free" anyons are studied in a two-dimensional spacetime since no $(2+1)$-dimensional model of free anyons can exist ${ }^{1}$ [17. In the setting of CAR algebras on the fermionic Fock space there exists a natural notion of second quantization more appropriate for a theory of relativistic

\footnotetext{
${ }^{1}$ This notion of "free" anyons refers to the on-mass-shell nature of the Fourier transform. In $\mathrm{d}=1+1$ the anyon operators can live together in the same Hilbert space as the free Fermions, but they are not really free in the mass-shell sense.
} 
particles. The theory of Fermionic gauge groups [5, 6] displays a wide class of unitarily implementable automorphisms on the antisymmetric Fock space.

Our choice is the natural one [1, i.e. that for which the winding numbers (the charges) are easily computable through index formulae [21], while the zero charge implementers are the well known smeared-out kink operators since they can modify the statistics of a sector [22]. Implementable gauge groups in the one-particle Dirac theory lead to a model which exhibits strange statistics. A class of Bogoliubov automorphisms unitarily implementable in the Fock representation induce a family of localized and transportable automorphisms of the observable algebra [1], implemented by non local operators which are not even contained in the field algebra $\mathscr{F}$.

Since our investigations are strongly influenced by the violation of Haag duality and the non locality of implementers, which gives rise to non inner automorphisms, we begin with a discussion of the arguments leading to the known results, in order to emphasize the need to clarify the notion of statistics even for theories not fulfilling all the axioms of AQFT, and to better understand the developments presented in this paper. Statistics of sectors, approached first with "classical" DHR theory [7, 8, 9, 10, 12, 13, depends not only on the charge (i.e. on the sector), but also on a continuous parameter which indexes a collection of unitarily implementable automorphisms which carry no charge, but modify the statistics of the composed sector [22].

Unfortunately, since the net of local observables does not fulfill Haag duality, some results largely exploited in AQFT are no longer true in the setting with which we are concerned here, and we are able to produce counterexamples. However, the statistics operator still possesses all of the formal properties as it does in DHR theory, since unitary intertwiners between automorphisms of the same translation equivalent class are always local elements of $\mathscr{A}$, even if Haag duality is violated.

After computing the statistics operator formally, the question remains as to whether the braiding obtained in this way has a genuine meaning in terms of statistics. Actually, we cannot proceed step by step along DHR theory alone, as it deals with local objects and often exploits Haag duality as a fundamental technical assumption. We are now analyzing a theory that allows for intertwiners not lying in the algebra where endomorphisms act and where endomorphisms are not locally inner but are inner only in a asymptotical sense.

We appeal to a more recent notion of braiding [2], where the condition of asymptotic abelianness of intertwiners allow us to define bi-asymptopias, giving rise to a braiding for a suitable full subcategory of $\operatorname{End}(\mathscr{A})$. In higher dimensions, Roberts has shown that a DHR sector of a non-Haag dual net $\mathscr{A}$ extends to a DHR sector of the dual net $\mathscr{A}^{d}$, and the latter can be studied with the usual methods [19]. In $(1+1)$-dimensional massive theories this fails since $\mathscr{A}^{d}$, satisfying Haag duality and split property for wedges, has no localized sectors as Mueger has shown in [15]. The net $\mathscr{A}$ may have 
non-trivial localized sectors, but they necessarily become solitons when they are extended to $\mathscr{A}^{d}$.

In $(1+1)$-dimensional free massive Dirac field theory we exclude those braidings that do not give rise to true statistics, since they have their DHR counterpart in pseudo statistics operators constructed without remaining in the same connected component. Bi-asymptopias relative to different components are not mutually cofinal, nor path connected, due to the geometry of a two-dimensional space-time. A direct computation for each connected component yields different braidings, i.e. the category is not symmetric. Physically speaking, particles described by this theory are neither bosons nor fermions for almost all values of the solitonic parameter $\lambda$.

The present article is organized as follows. In Sect. 2 our assumptions are stated: relativistic second quantization, implementable gauge groups in the $(1+1)$-dimensional free massive Dirac field theory, index formulae for smeared-out kink operators in the formalism developed in [6], [5], 21].

In Sect. 3 we analyze a field theory model arising from the fermionic gauge group theory when applied to the Dirac field in two-dimensional Minkowski space. Some known results from [1] are derived. Investigation of the statistics in the framework of DHR analysis leads to anomalous behaviour of the charge composition, and statistics is not an intrinsic property of the sector ${ }^{2}$.

In Sect. 4 we prove that unitary implementers are not elements of the global field algebra $\mathscr{F}$ for almost all values of the continuous parameter $\lambda$, thus giving a complete classification of their localization properties.

In Sect. 5 we prove asymptotic abelianness in order to exhibit a pair of disjoint (i.e not path connected) bi-asymptopias which give rise to an authentic braiding for the $C^{*}$-subcategory of $\operatorname{End}(\mathscr{A})$ generated by our family $\Delta$ of automorphisms. We compute the braiding explicitly and give a natural condition to be imposed in (1+1)-dimension in order to avoid those braidings with no true counterpart in the DHR setting.

\section{Preliminaries}

Since we work in the algebraic setting, we briefly list the axioms appropriate for theories where observables are defined from fields through a principle of gauge invariance.

1. The field algebra $\mathscr{F}$ is the inductive limit of the net of von Neumann algebras $\mathcal{O} \rightarrow \mathscr{F}(\mathcal{O})$ and its action on the Hilbert space $\mathscr{H}$ is irreducible.

\footnotetext{
${ }^{2}$ In the conformally invariant zero mass (or short distance) limit the situation changes radically and the standard DHR theory becomes again fully applicable. This phenomenon is inexorably linked to the emergence of new sectors in this limit (the disorder becomes charge-carrying) and has been observed in [14]
} 
2. There exists a strongly continuous unitary representation $L \rightarrow \mathcal{U}(L)$ of the Poincaré group $\mathscr{P}$ on $\mathscr{H}$ inducing automorphisms $\alpha_{L}$ of the field algebra, and the action on local algebras is geometric, i.e. $\alpha_{L}(\mathscr{F}(\mathcal{O}))=$ $\mathscr{F}(L \mathcal{O})$. Moreover, there exists a unit vector $\Omega \in \mathscr{H}$, the vacuum vector, unique up to a phase, which is left invariant by $\mathcal{U}(L), L \in \mathscr{P}$. The vector state induced by $\Omega$ is the vacuum state $\omega_{0}$ of $\mathscr{F}$,

$$
\omega_{0}(F)=(\Omega, F \Omega)
$$

3. (Reeh-Schlieder property for double cones) The vacuum vector $\Omega$ is cyclic and separating for every algebra $\mathscr{F}(\mathcal{O})$.

4. There exists a faithful representation $g \rightarrow \beta_{g}$ of a compact group $G$, the gauge group, by automorphisms of $\mathscr{F} . \beta_{g}$ commutes with $\alpha_{L}$ and $\beta_{g}(\mathscr{F}(\mathcal{O}))=\mathscr{F}(\mathcal{O})$. Moreover, for $F \in \mathscr{F}(\mathcal{O})$, the correspondence $g \rightarrow \beta_{g}(F)$ is weakly continuous.

5. (Normal commutation relations) There exists a $k \in G$, with $k^{2}=e$, such that, setting

$$
\mathscr{F}_{ \pm}(\mathcal{O})=\left\{F \in \mathscr{F}(\mathcal{O}): \beta_{k}(F)= \pm F\right\},
$$

we have that $\mathscr{F}_{+}\left(\mathcal{O}_{1}\right)$ commutes with $\mathscr{F}\left(\mathcal{O}_{2}\right)$ and $\mathscr{F}_{-}\left(\mathcal{O}_{1}\right)$ anticommutes with $\mathscr{F}_{-}\left(\mathcal{O}_{2}\right)$ when $\mathcal{O}_{1} \subset \mathcal{O}_{2}^{\prime}$. If the unitary $\Gamma(g)$ implements the automorphism $\beta_{g}$, we can reformulate (II) by requiring twisted locality:

$$
\mathscr{F}(\mathcal{O})^{\tau} \subset \mathscr{F}\left(\mathcal{O}^{\prime}\right)^{\prime}
$$

where $\mathscr{F}(\mathcal{O})^{\tau}:=Z \mathscr{F}(\mathcal{O}) Z^{*}, Z=\frac{\mathbb{1}+i \Gamma(k)}{1+i}$, defines the twisted algebra.

\subsection{Relativistic second quantization}

We now fix notation and give an overview of the fundamentals of relativistic second quantization. Let $\mathscr{H}$ be a separable complex Hilbert space with inner product $($,$) . The fermionic Fock space \mathcal{F}_{a}(\mathscr{H})$ is the completion of the vector space $\mathscr{D}_{a t}:=\bigoplus_{n=0}^{\infty} \wedge^{n} \mathscr{H}$ of antisymmetric algebraic tensors with respect to the "natural" scalar product $<\oplus_{n \geqslant 0} \xi_{n} \mid \oplus_{n \geqslant 0} \eta_{n}>=\sum_{n \geqslant 0}\left(\xi_{n}, \eta_{n}\right)$, with the standard unity vector $\Omega:=(1,0,0, \ldots)$ defined as the vacuum vector. For every $f \in \mathscr{H}$, the creation operator $c^{*}(f)$ and its adjoint $c(f)$, the annihilation operator, are defined on the whole fermionic Fock space, with $\left\|c^{*}(f)\right\|=\|f\|$, and they satisfy the canonical anticommutation relations:

$$
\begin{gathered}
\{c(f), c(g)\}=\left\{c^{*}(f), c^{*}(g)\right\}=0 \\
\left\{c(f), c^{*}(g)\right\}=(f, g) \mathbb{1}
\end{gathered}
$$


for arbitrary $f, g \in \mathscr{H}$. If $U \in \mathcal{B}(\mathscr{H})$, we define the operator $\Gamma(U)$ on $\overline{\wedge^{n} \mathscr{H}}$ by

$$
\Gamma(U) c^{*}\left(f_{1}\right) \cdots c^{*}\left(f_{n}\right) \Omega=c^{*}\left(U f_{1}\right) \cdots c^{*}\left(U f_{n}\right) \Omega,
$$

which has the property $\Gamma(U) \Gamma(V)=\Gamma(U V)$. In particular, if $U \in \mathcal{U}(\mathscr{H}):=$ $\{A: \mathscr{H} \rightarrow \mathscr{H}, A$ unitary $\}$, the correspondence $c^{*}(f) \mapsto c^{*}(U f)$ is an automorphism of the CAR algebra, unitarily implemented by $\Gamma(U)$ (in the Fock representation):

$$
\Gamma(U) c^{*}(f) \Gamma(U)^{*}=c^{*}(U f)
$$

as follows from (2). Moreover, an arbitrary $A \in \mathcal{B}(\mathscr{H})$ induces on $\mathcal{F}_{a}(\mathscr{H})$ a sum operator $\mathrm{d} \Gamma(A)$ such that $\exp (i t \mathrm{~d} \Gamma(A))=\Gamma\left(e^{i t A}\right)$, which preserves the adjoint and the commutator [5].

In concrete cases, as that we discuss in this paper, $\mathscr{H}=\mathscr{H}_{+} \oplus \mathscr{H}_{-}$, where $\mathscr{H}_{ \pm}$are copies of the same function space $L^{2} \equiv H$. Let $P_{\delta}(\delta=+,-)$ be the projectors onto $\mathscr{H}_{ \pm}$. If $A$ is an operator on $\mathscr{H}$, we set $A_{\delta \delta^{\prime}}:=$ $P_{\delta} A P_{\delta^{\prime}}, \delta, \delta^{\prime}=+,-$. In this notation, $A$ is a block matrix whose entries are endomorphisms of $L^{2}$. Such a decomposition of $\mathscr{H}$ is related to the well known fact that the free Dirac hamiltonian $H_{m}$ has spectrum $(-\infty,-m] \cup$ $[m,+\infty)$, where $m \geqslant 0$ denotes the rest mass of the particle. Here, $P_{ \pm}$ are the spectral projectors of $H_{m}$ onto $[m,+\infty)$ and $(-\infty,-m]$ respectively. Instead of non-relativistic second quantization $A \mapsto \mathrm{d} \Gamma(A)$, we work with another irreducible representation of the CAR algebra, defined by

$$
\tilde{c}(f):=c\left(P_{+} f\right)+c^{*}\left(\overline{P_{-} f}\right) .
$$

The one-particle Hilbert space is then defined by $\mathscr{H}_{1}:=P_{+} H \oplus \overline{P_{-} H}$ while the physical Hilbert space is $\mathcal{F}_{a}\left(\mathscr{H}_{1}\right)$. If $U \in \mathcal{U}(\mathscr{H})$ satisfies $\left[U, P_{\delta}\right]=0$, then the automorphism $\tilde{c}(f) \mapsto \tilde{c}(U f)$ is unitarily implemented by

$$
\widetilde{\Gamma}(U):=\Gamma\left(U_{++}\right) \Gamma\left(\bar{U}_{--}\right),
$$

with the compact notation $\Gamma\left(U_{++}\right) \equiv \Gamma\left(U_{++} \oplus \mathbb{1}\right), \Gamma\left(\bar{U}_{--}\right) \equiv \Gamma\left(\mathbb{1} \oplus \bar{U}_{--}\right)$. Here the bar over an operator stands for the action by a fixed conjugation $J$ on $\mathscr{H}$, i.e. $\bar{U}=J U J$. For arbitrary $U \in \mathcal{U}(\mathscr{H})$, the Shale-Stinespring theorem states that there exists a unitary $\widetilde{\Gamma}(U)$ on the antisymmetric Fock space such that

$$
\tilde{c}(U f)=\widetilde{\Gamma}(U) \tilde{c}(f) \widetilde{\Gamma}(U)^{*} \quad \forall f \in \mathscr{H}
$$

if and only if the off-diagonal parts of $U$, namely $U_{\delta-\delta}$, are Hilbert-Schmidt $(H S)$ operators. Unitaries on $\mathscr{H}$ inducing automorphisms of the CAR algebra which are unitarily implementable on $\mathcal{F}_{a}(\mathscr{H})$ form a group, denoted $\mathcal{G}_{2}$. Let $\mathfrak{g}_{2}:=\left\{A \in \mathcal{B}(\mathscr{H}): A_{\delta,-\delta} \in H S\right\}$ be the complex Lie algebra of $\mathcal{G}_{2}$. By a suitable choice for the phases of the unitary operator implementing the automorphism of the CAR algebra, it is always possible to define a one-parameter strongly continuous group

$$
\widetilde{\Gamma}\left(e^{i t A}\right)=e^{i t \mathrm{~d} \tilde{\Gamma}(A)}, \quad A=A^{*} \in \mathfrak{g}_{2}
$$


where $\mathrm{d} \tilde{\Gamma}(A)$ is the self-adjoint generator. The arbitrary additive constant in the definition of $\mathrm{d} \tilde{\Gamma}(A)$ is fixed by requiring that $(\Omega, \mathrm{d} \tilde{\Gamma}(A) \Omega)=0$. In Sect. 3 , where we deal with the Dirac field, we shall employ the more common notation $\pi(\phi(f))$ rather than $\tilde{c}(f)$.

Definition 1 The charge operator $Q$ is the generator of the one-parameter group induced by the identity:

$$
Q:=\mathrm{d} \widetilde{\Gamma}(\mathbb{1})=\mathrm{d} \Gamma\left(P_{+}\right)-\mathrm{d} \Gamma\left(P_{-}\right) .
$$

Under the action of charge operator, fermionic Fock space splits into a direct sum of charge sectors $\mathcal{F}_{a}(\mathscr{H})=\bigoplus_{n \in \mathbb{Z}} \mathcal{F}_{n}$, and

$$
\widetilde{\Gamma}(U) \mathcal{F}_{n}=\mathcal{F}_{n+q(U)}, \quad U \in \mathcal{G}_{2},
$$

where $q(U) \in \mathbb{Z}$ is the Fredholm index of $U_{--}$.

The additive property $q\left(U_{1} U_{2}\right)=q\left(U_{1}\right)+q\left(U_{2}\right)$ also holds. In other terms, if $q(U)=1$, the vacuum sector $\mathcal{F}_{0}$ can be connected to the $n$ charge sector by applying $\widetilde{\Gamma}(U)^{n}$, while $q\left(e^{i t A}\right)=0, \quad A=A^{*} \in \mathfrak{g}_{2}$, since $q\left(e^{i t A}\right)=$ $q(\mathbb{1})=0$ by virtue of the continuity in $t$. Hence the charged sectors are left invariant by $\widetilde{\Gamma}\left(e^{i t A}\right)$. We end this overview with an identity of great relevance for computations:

$$
\Gamma(-\mathbb{1}) \widetilde{\Gamma}(U)=(-1)^{q(U)} \widetilde{\Gamma}(U) \Gamma(-\mathbb{1}) .
$$

In view of the subsequent applications, we cite three useful propositions which establish the commutation rules between unitary implementers and their self-adjoint generators [5].

Proposition 1 For every $A, B \in \mathfrak{g}_{2}$, on the domain $\mathscr{D}$ of finite particle vectors there holds

$$
[\mathrm{d} \widetilde{\Gamma}(A), \mathrm{d} \widetilde{\Gamma}(B)]=\mathrm{d} \tilde{\Gamma}([A, B])+C(A, B) \mathbb{1},
$$

where $C(A, B):=\operatorname{Tr}\left(A_{-+} B_{+-}-B_{-+} A_{+-}\right)$is the Schwinger term.

Here $\mathscr{D}:=\left\{F \in \mathcal{F}_{a}(\mathscr{H}): F=P_{l} F\right.$, for some $\left.l \in \mathbb{N}\right\}$ and $P_{l}$ denotes the spectral projector of the particle number operator on $[0, l]$.

Proposition 2 Let $A, B \in \mathfrak{g}_{2}, A=A^{*}, B=B^{*}$ and $[A, B]=0$. We have:

$$
\widetilde{\Gamma}\left(e^{i A}\right) \widetilde{\Gamma}\left(e^{i B}\right)=e^{-C(A, B) / 2} \widetilde{\Gamma}\left(e^{i(A+B)}\right) .
$$

The third proposition establishes the commutation rule between second quantization operators in the case that one of them is a charge shift (i.e. it carries a non zero charge).

Proposition 3 Let $U \in \mathcal{G}_{2}, A=A^{*} \in \mathfrak{g}_{2},[U, A]=0$. Then

$$
\widetilde{\Gamma}\left(e^{i A}\right) \widetilde{\Gamma}(U)=e^{i(\tilde{\Gamma}(U) \Omega, \mathrm{d} \tilde{\Gamma}(A) \tilde{\Gamma}(U) \Omega)} \widetilde{\Gamma}(U) \widetilde{\Gamma}\left(e^{i A}\right) .
$$




\subsection{Implementable gauge groups in the one-particle Dirac theory}

In the theory of $(1+1)$-dimensional free massive Dirac field, gauge transformations are operators of multiplication by unitary matrices on $\check{\mathscr{H}} \equiv$ $L^{2}(\mathbb{R}, d x)^{\otimes 2}$, the image of $\mathscr{H} \equiv L^{2}(\mathbb{R}, d p)^{\otimes 2}$ by means of the Fourier transformation $\mathcal{F}$, employed to diagonalize the differential operator representing the free Dirac Hamiltonian. Since we are interested in lifting these unitaries to the Fock space, we must consider only gauge transformations which define unitaries in $\mathcal{G}_{2}$. We denote by $H_{1}(\mathbb{R})$ the Sobolev space, which consists of all absolutely continuous functions of $L^{2}(\mathbb{R})$ with derivatives in $L^{2}(\mathbb{R})$. Once we have introduced the group (under pointwise multiplication)

$$
L_{e} \mathrm{U}(1):=\left\{u \in \operatorname{Map}(\mathbb{R}, \mathrm{U}(1)) \mid u(\cdot)-1 \in H_{1}(\mathbb{R})\right\},
$$

we define two faithful unitary representations $\check{\pi}_{ \pm}$of $L_{e} \mathrm{U}(1)$ on $\check{\mathscr{H}}$, given by $\check{\pi}_{+}(u)=u(x) \oplus \mathbb{1}$ and $\check{\pi}_{-}(u)=\mathbb{1} \oplus u(-x)$, i.e. they act as multiplication by a function on one component space only. Then $\pi_{s}(u) \in \mathcal{G}_{2}$, and two projective unitary representations $\widetilde{\Gamma}\left(\pi_{ \pm}\right)$on $\mathcal{F}_{a}(\mathscr{H})$ are automatically defined.

Another global gauge transformation has the form $e^{i \varphi_{+}} \oplus e^{i \varphi_{-}}, \varphi_{ \pm} \in(0,2 \pi)$. In the case of interest to us, i.e. rest mass of the particle $m>0$, we put $\varphi_{+}=\varphi_{-}$, otherwise the $H S$ condition would be violated.

We end this paragraph with a formula for $\operatorname{Ind} U_{--}$. We are interested in operators of the form

$$
(\check{U} f)(x)=u(x) f(x), \quad u(x) \in \mathrm{U}(2), \quad f \in \check{\mathscr{H}},
$$

where the $2 \times 2$ matrix $u(x)$ is assumed to be diagonal:

$$
u(x)=\left(\begin{array}{cc}
u_{+}(x) & 0 \\
0 & u_{-}(x)
\end{array}\right), \quad u_{ \pm}(x) \in \mathbb{C} .
$$

We set $A:=\mathcal{F}^{-1} \check{A} \mathcal{F}$ for $\check{A}$ an operator on $\check{\mathscr{H}}$. We consider continuous multipliers of the form (9) such that, for each $s$, there exists $u_{\infty} \in C(\{ \pm 1\}, \mathrm{U}(1))$ satisfying

$$
u_{s}(x)-u_{\infty}\left(\frac{x}{|x|}\right)=o(1), \quad|x| \rightarrow \infty, \quad s=+,-.
$$

These multipliers form a group, denoted by $G_{h}$, and their Fredholm indices are easily computable in view of the following [21.

Theorem 1 Let $U \in G_{h}$. Then

$$
\text { Ind } U_{--}=\mathrm{w}\left(u_{+} u_{-}^{-1}\right) \text {, }
$$

where $\mathrm{w}$ is the winding number which, by convention, is positive on the map $x \rightarrow \frac{x-i}{x+i}$. 
In order to complete the discussion of the operators we shall employ in the next section, we remark that all our unitaries induce automorphisms of the CAR algebra which are unitarily implementable on the Fock space. Indeed, if $x_{1} \rightarrow \alpha\left(x_{1}\right)$ is an odd, monotonously increasing, $C^{\infty}$ real valued function, which equals 1 at the right of the interval $(-1,1)$, we introduce the smeared-out kink operators

$$
\check{U}_{\lambda, \epsilon}:=e^{i \pi \lambda \alpha(\cdot / \epsilon)}, \quad \lambda \in \mathbb{C}, \quad \epsilon>0 .
$$

The off-diagonal parts of $U_{\lambda, \epsilon}$ are $H S$ for every $\lambda \in \mathbb{C}$, so it induces Bogoliubov transformations unitarily implementable for every $\lambda \in \mathbb{R}[21]$.

\section{Strange statistics in two-dimensional free mas- sive Dirac field theory}

The theory of fermionic gauge group reveals itself as a natural setting for the construction of a model which exhibits anomalous statistics 1]. The initial Hilbert space is $L^{2}\left(\mathbb{R}, \mathbb{C}^{2}\right)$. Denoting by $\mathcal{K}$ the set of double cones in the $(1+1)$-dimensional Minkowski space, let $B_{\mathcal{O}}$ be the base at time $t$ of

$\mathcal{O} \in \mathcal{K}$. The algebra of fields localizable in $\mathcal{O}$ is defined in the usual way,

$$
\mathscr{F}(\mathcal{O})=\left\{\pi\left(\phi\left(e^{i t H_{m}} f\right)\right): \operatorname{supp}(f) \subset B_{\mathcal{O}}\right\}^{\prime \prime},
$$

and the global field algebra $\mathscr{F}$ is the $C^{*}$-inductive limit of the net $\{\mathscr{F}(\mathcal{O})\}_{\mathcal{O} \in \mathcal{K}}$. The gauge invariant parts (i.e. the subalgebras left invariant by $\operatorname{Ad} \tilde{\Gamma}\left(e^{i \gamma}\right)$, $\gamma \in \mathbb{R})$ form the net of observables. Let us mention that the free massive Dirac field theory in $(1+1)$-dimensions fulfills twisted duality $\mathscr{F}(\mathcal{O})^{\tau}=$ $\mathscr{F}\left(\mathcal{O}^{\prime}\right)^{\prime}$ (the proof of this is independent of the space-time dimension; see [4] for details), but the net of observables does not fulfil Haag duality for double cones. Indeed, if $\mathcal{O}, \mathcal{O}_{1}, \mathcal{O}_{2}$ are double cones, with bases at $t=0$, such that $\mathcal{O}_{1}, \mathcal{O}_{2}$ lie in different connected components of $\mathcal{O}^{\prime}$, and if $f_{i}$ are test functions with $\operatorname{supp}\left(f_{i}\right) \subset \mathcal{O}_{i}(i=1,2)$, then the observable $\pi\left(\phi\left(f_{1}\right)\right)^{*} \pi\left(\phi\left(f_{2}\right)\right)$ is contained in $\mathscr{A}(\mathcal{O})^{\prime}$ but not in $\mathscr{A}\left(\mathcal{O}^{\prime}\right)^{\prime \prime}$ [1, 16.

The automorphism of $\mathscr{F}$ defined by $\rho_{Z}(\pi(\phi(f))):=\pi(\phi(Z f))$ is unitarily implemented in the Fock representation when $Z$ is one of the following unitaries of $L^{2}\left(\mathbb{R}, \mathbb{C}^{2}\right)$ :

$$
\begin{gathered}
(U(n) f)(x)=\left(e^{i \pi n \varepsilon(x)} f_{1}(x), f_{2}(x)\right), \quad n \in \mathbb{Z} \\
(V(\lambda) f)(x)=e^{i \pi \lambda \vartheta(x)} f(x), \quad \lambda \in \mathbb{R},
\end{gathered}
$$

which correspond in (9) to the choice $u_{+}(x)=\exp (i \pi n \varepsilon(x)), u_{-} \equiv 0$ and $u_{ \pm}(x)=\exp (i \pi \lambda \vartheta(x))$, respectively. Here, the functions $\varepsilon$ and $\vartheta$ are characterized by the same properties of $\alpha$ relative to generic intervals $(-\epsilon, \epsilon)$, resp. $(-\theta, \theta)$, instead of $(-1,1)$. We emphasize that this result is valid only in the 
massive case [18. The gauge group $\mathrm{U}(1)$ acts on $\mathscr{F}$ through $e^{i \gamma} \mapsto \operatorname{Ad} \tilde{\Gamma}\left(e^{i \gamma}\right)$, and the self-adjoint unitary operator inducing the twisting is $\tilde{\Gamma}(-\mathbb{1})$.

We refine Propositions 1 and 2 to suit our purpose [18. The computation of the statistics operator needs commutation rules between implementers when translated to mutually space-like regions. Let $\mathcal{O}$ be a double cone with basis $(-\epsilon, \epsilon)$ at $t=0$, and $x, x^{\prime} \in \mathbb{R}$ such that $\mathcal{O}+x^{\prime} \subset(\mathcal{O}+x)^{\prime}$. Since the Schwinger term for the pair $V(\lambda)_{x}, V\left(\lambda^{\prime}\right)_{x^{\prime}}$ vanishes when $\mathcal{O}+x^{\prime} \subset$ $(\mathcal{O}+x)^{\prime}$, Proposition 2 establishes that the projective representation $\tilde{\Gamma}$ is multiplicative in almost all cases of interest to us. Proposition 3 applies to the case $U=U(n)_{x}$ and $e^{i A}=V(\lambda)_{x^{\prime}}$, giving

$$
\begin{gathered}
\tilde{\Gamma}\left(V(\lambda)_{x^{\prime}}\right) \tilde{\Gamma}\left(U(n)_{x}\right)=e^{i \pi n \lambda \operatorname{sgn}\left(x-x^{\prime}\right)} \tilde{\Gamma}\left(U(n)_{x}\right) \tilde{\Gamma}\left(V(\lambda)_{x^{\prime}}\right), \\
\tilde{\Gamma}\left(U(n)_{x}\right) \tilde{\Gamma}\left(U\left(n^{\prime}\right)_{x^{\prime}}\right)=\tilde{\Gamma}\left(U\left(n^{\prime}\right)_{x^{\prime}}\right) \tilde{\Gamma}\left(U(n)_{x}\right) .
\end{gathered}
$$

As will be clear later, we consider only even charge $n$, while the real number $\lambda$ is left arbitrary. Since $\mathcal{G}_{2}$ is a group, the product $U(n) V(\lambda)$ is unitarily implementable too. We set $W \equiv W(n, \lambda):=U(n) V(\lambda)$. Choosing the same generating function $\vartheta=\varepsilon$ we obtain a unitary operator on $L^{2}\left(\mathbb{R}, \mathbb{C}^{2}\right)$ defined by:

$$
W:=e^{i \pi(n+\lambda) \varepsilon(\cdot)} \oplus e^{i \pi \lambda \varepsilon(\cdot)} .
$$

Here $W$ acts on both components of $L^{2}\left(\mathbb{R}, \mathbb{C}^{2}\right)$ as multiplication by two distinct functions. We note that $U(n) \equiv W(n, 0)$ and $V(\lambda) \equiv W(0, \lambda)$. In order to determine the charge carried by the automorphism $\rho_{W}$ we must evaluate $q(W)$. The Fredholm index of $W_{--}$can be easily computed as an immediate application of Theorem 11 and it equals $n$. Note that $V(\lambda)$ belongs to the connected component of the identity, therefore $\operatorname{Ind} V(\lambda)=\operatorname{Ind}(\mathbb{1})=0$, $\lambda \in \mathbb{R}$. Thus $n$ is the charge carried by $W$, with no contribution from $V(\lambda)$. (The charge is entirely transported by $U(n)$ while $V(\lambda)$ is neutral).

Proposition 4 Automorphisms of $\mathscr{F}$ of the form $\rho_{W}$ induce, on restriction, automorphisms of the observable algebra $\mathscr{A}$.

Proof. Since any $*$-homomorphism between $C^{*}$ algebras is continuous, the statement is an immediate consequence of the inclusion

$$
\rho_{W}\left(\mathscr{A}\left(\mathcal{O}_{1}\right)\right) \subset \mathscr{A}\left(\mathcal{O}_{1}\right), \quad \forall \mathcal{O}_{1} \in \mathcal{K} .
$$

In order to prove this, let us consider a unitary $W(n, \lambda)$ with generating function $\varepsilon$ centred in $\mathcal{O} \in \mathcal{K}$. (We assume that all double cones have base at $t=0)$. We note that $\rho_{W}\left(\mathscr{F}\left(\mathcal{O}_{1}\right)\right) \subset \mathscr{F}\left(\mathcal{O}_{1}\right)$. Indeed, if $\operatorname{supp}(f) \subset B_{\mathcal{O}_{1}}$, then $\operatorname{supp}(W f) \subset B_{\mathcal{O}_{1}}$ too, hence :

$$
\rho_{W}(\pi(\phi(f))) \equiv \pi(\phi(W f)) \in \mathscr{F}\left(\mathcal{O}_{1}\right) .
$$

Moreover, $\left[\rho_{W}(A), \widetilde{\Gamma}\left(e^{i \gamma}\right)\right]=0$ for all $A \in \mathscr{A}\left(\mathcal{O}_{1}\right)$ and $\gamma \in \mathbb{R}$, since the adjoint actions $\operatorname{Ad} \tilde{\Gamma}\left(e^{i \gamma}\right)$ and $\operatorname{Ad} \tilde{\Gamma}(V)$ commute between themselves in view of Proposition 3. The claim follows from the gauge invariance of $A$. 
Proposition 5 Automorphisms of $\mathscr{A}$ defined as in Proposition 4 are localizable in double cones.

Proof. Let $A:=\pi(\phi(f)), \operatorname{supp}(f) \subset B_{\mathcal{O}_{1}}$, where $\mathcal{O}_{1} \subset \mathcal{O}^{\prime}$. Obviously, if $x \notin \operatorname{supp}(f)$ then $(W f)(x)=0$. If $x \in \operatorname{supp}(f) \subset B_{\mathcal{O}_{1}}$, then $x \notin B_{\mathcal{O}}$ and $\varepsilon(x)= \pm 1$, and we have

$$
(W f)(x)=e^{ \pm \pi i \lambda} f(x),
$$

hence $\rho_{W}(A)=\operatorname{Ad} \tilde{\Gamma}\left(e^{ \pm \pi i \lambda}\right)(A), \forall A \in \mathscr{F}\left(\mathcal{O}_{1}\right)$. Now, it is then evident that, if $A \in \mathscr{A}\left(\mathcal{O}_{1}\right) \equiv \mathscr{F}\left(\mathcal{O}_{1}\right)^{\mathrm{U}(1)}$, then $\rho_{W}(A)=A$, i.e. $\rho_{W}$ acts trivially on $\mathscr{A}\left(\mathcal{O}^{\prime}\right)$.

Proposition 6 For each unitary $W$ defined as above, $\rho_{W}:=\operatorname{Ad} \widetilde{\Gamma}(W)$ defines a localizable and translatable automorphism of $\mathscr{A}$.

Proof. We have just proved localizability: $\left.\rho_{W}\right|_{\mathscr{A}\left(\mathcal{O}^{\prime}\right)}=\left.\mathrm{id}\right|_{\mathscr{A}\left(\mathcal{O}^{\prime}\right)}$, where $\mathcal{O}$ is the double cone in whose base the unitary $W$ (i.e. its generating function $\varepsilon$ ) is "centred", and $\rho_{W}(\mathscr{A}(\tilde{\mathcal{O}})) \subset(\mathscr{A}(\tilde{\mathcal{O}}))$ for every double cone $\tilde{\mathcal{O}} \supset \mathcal{O}$. In order to prove translatability, let us observe that denoting the translates by $W_{x}:=T(x) W T(-x)$, the automorphism $\rho_{W_{x}}$ is localized in $\mathcal{O}+x$. Moreover, the unitary $\widetilde{\Gamma}\left(W_{x} W^{*}\right)$ intertwines $\rho_{W}:=\operatorname{Ad} \tilde{\Gamma}(W)$ and $\rho_{W_{x}}:=\operatorname{Ad} \tilde{\Gamma}\left(W_{x}\right)$, and induces an equivalence between them since it is a local observable. Indeed, $\widetilde{\Gamma}\left(W_{x} W^{*}\right) \in \mathscr{A}(\tilde{\mathcal{O}})$, with $\tilde{\mathcal{O}} \supset \mathcal{O} \cup \mathcal{O}_{x}$. The gauge invariance comes from the commutation relation of Proposition 3 between $\widetilde{\Gamma}(V)$ and $\widetilde{\Gamma}\left(e^{i A}\right)$, where the inner product (8) now vanishes. Indeed, since $q\left(W_{x} W^{*}\right)=q\left(W_{x}\right)+q\left(W^{*}\right) \equiv q(W)-q(W)=0$, it follows that the intertwiner $\widetilde{\Gamma}\left(W_{x} W^{*}\right)$ preserves the charge and so $\widetilde{\Gamma}\left(W_{x} W^{*}\right) \Omega \in \mathbb{C} \Omega$. We thus have:

$$
\left(\widetilde{\Gamma}\left(W_{x} W^{*}\right) \Omega, \mathrm{d} \widetilde{\Gamma}(\gamma \mathbb{1}) \widetilde{\Gamma}\left(W_{x} W^{*}\right) \Omega\right)=(\Omega, \mathrm{d} \widetilde{\Gamma}(\gamma \mathbb{1}) \Omega)=0 .
$$

In order to prove that $\widetilde{\Gamma}\left(W_{x} W^{*}\right) \in \mathscr{F}\left(\tilde{\mathcal{O}}^{\prime}\right)^{\prime}$, let us consider $\mathcal{O}_{1} \subset \tilde{\mathcal{O}}^{\prime}$ and $\operatorname{supp}(f) \subset B_{\mathcal{O}_{1}}$. In order to evaluate the expression

$$
\widetilde{\Gamma}\left(W_{x} W^{*}\right) \pi(\phi(f)) \widetilde{\Gamma}\left(W_{x} W^{*}\right)^{*}=\rho_{W_{x}} \circ \rho_{W^{*}}(\pi(\phi(f))),
$$

we notice that $\rho_{W^{*}} \equiv \rho_{W}^{-1}$ is still localized in $\mathcal{O}$, and then, since $W(n, \lambda)^{*}=$ $W(-n,-\lambda)$,

$$
\rho_{W^{*}}(\pi(\phi(f)))=\pi\left(\phi\left(e^{-i \pi \lambda} f\right)\right) .
$$

Analogously

$$
\rho_{W_{x}}(\pi(\phi(f)))=\pi\left(\phi\left(e^{i \pi \lambda} f\right)\right)
$$


so the right side of (12) reduces to $\pi(\phi(f))$ and the result follows from twisted duality.

In the previous proof we have incidentally established that unitaries $\tilde{\Gamma}(W)$ are gauge invariant if and only if $q(W)=0$.

We are now in position to perform the computation of the statistics operator $\varepsilon_{\rho_{W}}$. For simplicity, we start with an automorphism $\rho_{W}$ localized in a double cone $\mathcal{O}$ centred at the origin.

Proposition 7 If the automorphism $\rho_{W}$ is localized in a double cone centred at the origin, its statistics operator is

$$
\varepsilon_{\rho_{W}}=e^{ \pm 2 \pi i n \lambda} \mathbb{1}
$$

according to the connected component of $\mathcal{O}^{\prime}$.

Proof. Since we work at $t=0$, we omit the component-subscript and consider $x \in \mathbb{R}$. The automorphism $\rho_{W_{x}}$ is localized in $\mathcal{O}+x$ and is unitarily equivalent to $\rho_{W}$ through the intertwiner $\tilde{\Gamma}\left(W_{x} W^{*}\right)$. Then,

$$
\varepsilon_{\rho_{W}}=\widetilde{\Gamma}\left(W_{x} W^{*}\right)^{*} \rho_{W}\left(\widetilde{\Gamma}\left(W_{x} W^{*}\right)\right)=\widetilde{\Gamma}(W) \widetilde{\Gamma}\left(W_{x}\right)^{*} \widetilde{\Gamma}(W) \widetilde{\Gamma}\left(W_{x}\right) \widetilde{\Gamma}\left(W^{*}\right)^{2} .
$$

(Here, and in the sequel, we omit all cocyles since they are always coupled with their conjugate). With this convention the previous expression yields

$$
\widetilde{\Gamma}(W) \widetilde{\Gamma}\left(W_{x}^{*}\right) \widetilde{\Gamma}(U) \widetilde{\Gamma}(V) \widetilde{\Gamma}\left(U_{x}\right) \widetilde{\Gamma}\left(V_{x}\right) \widetilde{\Gamma}(V)^{*} \widetilde{\Gamma}(U)^{*} \widetilde{\Gamma}(W)^{*} .
$$

By Proposition [3] and our remarks on the specific cases discussed in [18],

$$
\begin{aligned}
\widetilde{\Gamma}(V) \widetilde{\Gamma}\left(U_{x}\right) \equiv \widetilde{\Gamma}\left(e^{i X(\lambda)}\right) \widetilde{\Gamma}\left(U(n)_{x}\right) & =e^{i\left(\tilde{\Gamma}\left(U_{x}\right) \Omega, \mathrm{d} \tilde{\Gamma}(X(\lambda)) \tilde{\Gamma}\left(U_{x}\right) \Omega\right)} \widetilde{\Gamma}\left(U_{x}\right) \widetilde{\Gamma}\left(e^{i X(\lambda)}\right) \\
& =e^{i \pi n \lambda \operatorname{sgn}(x)} \widetilde{\Gamma}\left(U_{x}\right) \widetilde{\Gamma}(V),
\end{aligned}
$$

while $\widetilde{\Gamma}(V)$ commutes with $\widetilde{\Gamma}\left(V_{x}\right)$, and $\widetilde{\Gamma}\left(U_{x}\right)^{*}$ with $\widetilde{\Gamma}(U)$. We then obtain

$$
e^{i \pi n \lambda \operatorname{sgn}(x)} \widetilde{\Gamma}(W) \widetilde{\Gamma}\left(V_{x}^{*}\right) \widetilde{\Gamma}(U) \widetilde{\Gamma}\left(V_{x}\right) \widetilde{\Gamma}(U)^{*} \widetilde{\Gamma}(W)^{*} .
$$

Repeating the same arguments for the two central terms, one has

$$
\varepsilon_{\rho_{W}}=e^{2 \pi i n \lambda \operatorname{sgn}(x)} \mathbb{1}
$$

If the automorphism is translated to a double cone $\mathcal{O}+x$, for arbitrary $x$, it assumes the form $\rho_{W x}$, with $W$ localized around the origin. With a proof identical to that of Prop. [7 one easily obtains the following. 
Proposition 8 For every $x \in \mathbb{R}$,

$$
\varepsilon_{\rho_{W_{x}}}=e^{2 \pi i n \lambda \operatorname{sgn}(x-y)} \mathbb{1},
$$

with $\mathcal{O}+y$ the auxiliary double cone, spatially separated from $\mathcal{O}+x$, used in the construction of the statistics operator.

Remark. In view of the decomposition $\rho_{W}=\rho_{U} \rho_{V}$, an alternative method of evaluating $\varepsilon_{\rho_{W}}$ is based on the identity

$$
\varepsilon_{\rho_{U} \rho_{V}}=\rho_{U}\left(\varepsilon\left(\rho_{U}, \rho_{V}\right)\right) \varepsilon_{\rho_{U}} \rho_{U}^{2}\left(\varepsilon_{\rho_{V}}\right) \rho_{U}\left(\varepsilon\left(\rho_{V}, \rho_{U}\right)\right) .
$$

A straightforward computation reduces (13) to $\varepsilon_{\rho_{W}}=\rho_{U}\left(\varepsilon_{M}\left(\rho_{U}, \rho_{V}\right)\right)$, where the monodromy operator is simply $\varepsilon_{M}\left(\rho_{U}, \rho_{V}\right)=e^{2 \pi i n \lambda \operatorname{sgn}(y-x)} \mathbb{1}$.

We also observe that we could have determined the latter by exploiting the low-dimensional quantum field theory as formulated in [13], where only the statistics phases are involved. Indeed, since $\kappa_{W}=e^{2 \pi i n \lambda \operatorname{sgn}(y-x)}$ and $\kappa_{U}=\kappa_{V}=1$, the claim follows from

$$
\varepsilon\left(\rho_{V}, \rho_{U}\right) \varepsilon\left(\rho_{U}, \rho_{V}\right)=\frac{\kappa_{W}}{\kappa_{U} \kappa_{V}} \mathbb{1}
$$

[13. Lemma 3.3]. Therefore, these results are still consistent with the general theory of local quantum fields in low dimension.

We have incidentally noticed that in a $(1+1)$-dimensional massive QFT the statistics of a product may not coincide with the product of statistics, i.e. composition of DHR morphisms with ordinary statistics may generate braid statistics. This possibility is excluded by $(3+1)$-dimensional QFT with Haag duality [8, pag. 179], where $\varepsilon_{\xi_{1}} \varepsilon_{\xi_{2}}=\varepsilon_{\xi_{1} \xi_{2}}$ for two arbitrary superselection sectors $\xi_{1}, \xi_{2}$, (i.e. equivalence classes of Poincaré covariant localized automorphisms). The factorization property of statistics is no longer true in theories where non ordinary statistics can occur. In our model this property is equivalent to the triviality of monodromy, i.e. if and only if the automorphism carries ordinary statistics. Since unitary intertwiners between translation equivalent automorphisms are local observables, the violation of the multiplicative property of statistics cannot be attributed to the violation of Haag duality but to the geometry of space-time.

It is easily seen that the statistics operator depends on the translation equivalent class of automorphisms but not on its representative. The following Corollary is then evident.

Corollary 1 The statistics operator $\varepsilon_{\rho_{W}}$ gives rise to a one-dimensional representation of the braid group if and only if $2 n \lambda \notin \mathbb{Z}$.

We end this section with an expression for the statistics operator $\varepsilon\left(\rho_{W}, \rho_{W^{\prime}}\right)$ when the unitaries $W$ and $W^{\prime}$ are centred in the same interval. If the induced 
automorphisms are localized in $\mathcal{O}$, let $x, y \in \mathbb{R}^{2}$ be such that $\mathcal{O}_{x}$ and $\mathcal{O}_{y}$ lie in the right component of $\mathcal{O}^{\prime}$, with $\mathcal{O}_{x} \succ \mathcal{O}_{y}$, where by $\mathcal{O}_{x} \succ \mathcal{O}_{y}$ we mean that $\mathcal{O}_{x}$ lies in the right component of the space-like complement of $\mathcal{O}_{y}$. Let $\rho_{W_{x}}$ be localized in $\mathcal{O}_{x}$ and equivalent to $\rho_{W}$. Analogously, let $\rho_{W_{y}^{\prime}}$ be localized in $\mathcal{O}_{y}$ and equivalent to $\rho_{W^{\prime}}$. One then has

$$
\begin{aligned}
\varepsilon\left(\rho_{W}, \rho_{W^{\prime}}\right) & =\widetilde{\Gamma}\left(W^{\prime} W_{y}^{\prime *}\right) \times \widetilde{\Gamma}\left(W W_{x}^{*}\right) \circ \widetilde{\Gamma}\left(W_{x} W^{*}\right) \times \widetilde{\Gamma}\left(W_{y}^{\prime} W^{*}\right) \\
& =\widetilde{\Gamma}\left(W^{\prime} W_{y}^{\prime *}\right) \widetilde{\Gamma}\left(W_{y}^{\prime}\right) \widetilde{\Gamma}\left(W W_{x}^{*}\right) \widetilde{\Gamma}\left(W_{y}^{\prime}\right)^{*} \widetilde{\Gamma}\left(W_{x} W^{*}\right) \rho_{W}\left(\widetilde{\Gamma}\left(W_{y}^{\prime} W^{\prime *}\right)\right) \\
& =\widetilde{\Gamma}\left(W^{\prime} W_{y}^{\prime *}\right) \widetilde{\Gamma}\left(W_{y}^{\prime}\right) \widetilde{\Gamma}\left(W W_{x}^{*}\right) \widetilde{\Gamma}\left(W_{y}^{\prime}\right)^{*} \widetilde{\Gamma}\left(W_{y}^{\prime} W^{\prime *}\right) \widetilde{\Gamma}\left(W_{x} W^{*}\right) \\
& =e^{-i \pi\left(n \lambda^{\prime}+n^{\prime} \lambda\right)} \widetilde{\Gamma}\left(W^{\prime}\right) \widetilde{\Gamma}(W) \widetilde{\Gamma}\left(W^{\prime}\right)^{*} \widetilde{\Gamma}(W)^{*} \\
& =e^{-\pi i\left(n \lambda^{\prime}+n^{\prime} \lambda\right)} \mathbb{1} .
\end{aligned}
$$

On the other hand, if we choose $\mathcal{O}_{x} \prec \mathcal{O}_{y}$, the exponent in the last member changes sign. Therefore

$$
\varepsilon\left(\rho_{W}, \rho_{W^{\prime}}\right)=e^{i \pi\left(n \lambda^{\prime}+n^{\prime} \lambda\right) \operatorname{sgn}(y-x)} \mathbb{1} .
$$

\section{Charge implementers are not quasilocal}

As already stated, charge implementers $\tilde{\Gamma}(W)$ do not belong to the observable algebra. In this section we will show that they are not even in the field algebra $\mathscr{F}$ when $\lambda \neq 0 \bmod 2$. As a first step we observe that if $\tilde{\Gamma}(W) \in \mathscr{F}$, then its translates $\tilde{\Gamma}(W)_{x} \in \mathscr{F}$ too, through $\alpha_{x}(\pi(\phi(f)))=\pi\left(\phi\left(\tau_{x} f\right)\right)$, where $\left(\tau_{x} f\right)(\xi):=f(\xi-x)$. Once we have determined the statistics operator, we can exclude the trivial case $\lambda=0$ (no kinks present), since it yields ordinary statistics. Obviously, this is not the unique value of $\lambda$ for which the statistics reduce to the ordinary one. The other values which realize this possibility depend on the charge, since they are given by $2 n \lambda \in \mathbb{Z}$, and are trivially taken into account.

For $x \in \mathbb{R}$ such that $\mathcal{O}+x \subset \mathcal{O}^{\prime}$, we have

$$
\tilde{\Gamma}(W) \tilde{\Gamma}\left(W_{x}\right)=e^{2 \pi i q \lambda \operatorname{sgn}(x)} \tilde{\Gamma}\left(W_{x}\right) \tilde{\Gamma}(W),
$$

where $q \equiv q(W)=q\left(W_{x}\right)$ is the charge carried by both $\rho_{W}$ and $\rho_{W_{x}}$. For a general field $F$ we have

$$
F^{\tau}=F_{+}-i F_{-} \tilde{\Gamma}(-\mathbb{1})
$$

where $F_{ \pm}$denote the bosonic, resp. fermionic, part of $F$. This can be easily seen from the explicit form of the twisted field in the general case:

$$
F^{\tau}=\operatorname{Ad} Z(F), \quad Z=\frac{\mathbb{1}+i \tilde{\Gamma}(-\mathbb{1})}{1+i}
$$


(the symbol $\mathbb{1}$ always denotes the identity operator on the corresponding Hilbert space, as is clear from the context). We are interested in the case $2 q \lambda \notin \mathbb{Z}$, since this leads to a contradiction - the commutators are non vanishing, according to (15). Let $\tilde{\Gamma}(W) \in \mathscr{F}$. There exists a sequence of local fields $\left\{F_{n}\right\}_{n \in \mathbb{N}}$ norm convergent to $\tilde{\Gamma}(W)$, with $F_{n} \in \mathscr{F}\left(\mathcal{O}_{n}\right), n \in \mathbb{N}$. Since $\tilde{\Gamma}(W)^{\tau}=\tilde{\Gamma}(W)$, we have

$$
\left\|F_{n}^{\tau}-F_{n}\right\| \leqslant 2\left\|\tilde{\Gamma}(W)-F_{n}\right\| .
$$

On the other hand, according to (16), we have $F_{n}^{\tau}-F_{n}=-(1+i) F_{n}^{-} Z$, and thus $\left\|F_{n}^{\tau}-F_{n}\right\|=\sqrt{2}\left\|F_{n}^{-}\right\|$. By virtue of (17) we then have

$$
\left\|F_{n}^{-}\right\| \leqslant \sqrt{2}\left\|\tilde{\Gamma}(W)-F_{n}\right\|
$$

Let now $M>0$ be such that $\left\|F_{n}\right\|<M$. An $\varepsilon / 3$ argument yields

$$
\left\|\left[\tilde{\Gamma}(W), \tilde{\Gamma}(W)_{x}\right]\right\|<(M+1)\left\|\tilde{\Gamma}(W)-F_{n}\right\|+\left\|F_{n} F_{n, x}-\tilde{\Gamma}(W)_{x} \tilde{\Gamma}(W)\right\|,
$$

where we have used the fact that the translations, being implemented by unitary operators, are norm-preserving. Here $F_{n, x}$ denotes the translate of $F_{n}$ by $x$, for the generic $n \in \mathbb{N}$. The second term on the right hand side of (19) is dominated by

$$
\left\|F_{n} F_{n, x}-F_{n, x} F_{n}\right\|+\left\|F_{n, x} F_{n}-\tilde{\Gamma}(W)_{x} \tilde{\Gamma}(W)\right\| .
$$

Since $F_{n}$ and $F_{n, x}$ are local fields, $\left[F_{n}, F_{n, x}\right]=2 F_{n}^{-} F_{n, x}^{-}$when $\mathcal{O}_{n}+x \subset \mathcal{O}_{n}^{\prime}$. In this case we obtain, using (18):

$$
\left\|F_{n} F_{n, x}-F_{n, x} F_{n}\right\| \leqslant 2\left\|F_{n}^{-}\right\|\left\|F_{n, x}{ }^{-}\right\| \leqslant 4\left\|\tilde{\Gamma}(W)-F_{n}\right\|^{2} .
$$

In the last step we have exploited the commutativity between the actions $\alpha_{\mathbb{R}^{2}}$ and $\beta_{\mathrm{U}(1)}$ in order to yield $F_{x}{ }^{-}=F^{-}{ }_{x}$ for each quasilocal field $F$.

Let now $\varepsilon>0$, and let $n_{\varepsilon} \in \mathbb{N}$ be a positive integer such that

$$
\left\|\tilde{\Gamma}(W)-F_{n}\right\|<\frac{\varepsilon}{3(M+1)}, \quad\left\|F_{n, x} F_{n}-\tilde{\Gamma}(W)_{x} \tilde{\Gamma}(W)\right\|<\frac{\varepsilon}{3}
$$

for all $n \geqslant n_{\varepsilon}$. The integer $n_{\varepsilon}$ is independent of $x$. For $n=n_{\varepsilon}$, let $x>0$ be such that $\mathcal{O}_{n_{\varepsilon}}+x \subset \mathcal{O}_{n_{\varepsilon}}{ }^{\prime}$. Then, the left hand side of (19) can be made arbitrarily small, contradicting (15).

This proof, though intuitive, does not work if $2 q \lambda \in \mathbb{Z}$, since no contradiction is obtained in the latter case. In particular, our arguments exclude the case $q=0$. We will give an alternative proof which overcomes this impediment, based on twisted duality for the field algebra.

Theorem 2 For every $\lambda \neq 0 \bmod 2$ the unitary implementers $\tilde{\Gamma}(W)$ are not elements of the field algebra $\mathscr{F}$. If $\lambda=0 \bmod 2$, they are local elements of the field algebra. 
Proof. If $\widetilde{\Gamma}(W) \in \mathscr{F}$, let $\left\{F_{n}\right\}_{n \in \mathbb{N}}$ be a sequence of local elements of $\mathscr{F}$ norm convergent to $\widetilde{\Gamma}(W)$, with $F_{n} \in \mathscr{F}\left(\mathcal{O}_{n}\right)$ for suitable double cones $\mathcal{O}_{n}$. Since $F_{n}^{\tau} \in \mathscr{F}\left(\mathcal{O}_{n}\right)^{\tau}=\mathscr{F}\left(\mathcal{O}_{n}{ }^{\prime}\right)^{\prime},\left[F_{n}^{\tau}, A\right]=0, A \in \mathscr{F}\left(\mathcal{O}_{n}{ }^{\prime}\right), n \in \mathbb{N}$. If $\hat{\mathcal{O}}_{n} \subset \mathcal{O}_{n}{ }^{\prime}$, we have $\left[F_{n}^{\tau}, \pi(\phi(f))\right]=0, \operatorname{supp}(f) \subset B_{\hat{\mathcal{O}}_{n}}, n \in \mathbb{N}$. With this notation it follows that

$$
\begin{gathered}
\left\|\widetilde{\Gamma}(W)^{\tau} \pi(\phi(f))-\pi(\phi(f)) \widetilde{\Gamma}(W)^{\tau}\right\| \leqslant \\
\leqslant\left\|\left(\widetilde{\Gamma}(W)^{\tau}-F_{n}^{\tau}\right) \pi(\phi(f))\right\|+\left\|F_{n}^{\tau} \pi(\phi(f))-\pi(\phi(f)) F_{n}^{\tau}\right\|+ \\
+\left\|\pi(\phi(f))\left(F_{n}^{\tau}-\widetilde{\Gamma}(W)^{\tau}\right)\right\| \leqslant \\
\leqslant 2\|\pi(\phi(f))\|\left\|\widetilde{\Gamma}(W)^{\tau}-F_{n}^{\tau}\right\|+\left\|F_{n}^{\tau} \pi(\phi(f))-\pi(\phi(f)) F_{n}^{\tau}\right\| .
\end{gathered}
$$

Without lost of generality, we consider only normalized functions. Then, since the correspondence $f \rightarrow \pi_{P}(\phi(f))$ is isometric, $\|\pi(\phi(f))\|=1$. Fixing an arbitrary $\epsilon>0$, let $n_{\epsilon} \in \mathbb{N}$ be such that $\left\|\widetilde{\Gamma}(W)^{\tau}-F_{n}^{\tau}\right\|<\epsilon / 2$ for every $n \geqslant n_{\epsilon}$. For $n=n_{\epsilon}$ and $\operatorname{supp}(f) \subset B_{\hat{\mathcal{O}}_{n_{\epsilon}}}$, where $\hat{\mathcal{O}}_{n_{\epsilon}} \subset \mathcal{O}_{n_{\epsilon}}{ }^{\prime}$, the expression in the last line of (20) is less than $\epsilon$. Therefore, for each fixed $q$ and $\lambda$, $\left\|\widetilde{\Gamma}(W)^{\tau} \pi(\phi(f))-\pi(\phi(f)) \widetilde{\Gamma}(W)^{\tau}\right\|$ can be made arbitrary small by choosing a suitable function.

On the other hand, being $\widetilde{\Gamma}(W)^{\tau}=\widetilde{\Gamma}(W)$, the expression in the first line of (20) assumes the simpler form

$$
\left\|e^{-i \pi \lambda} \pi(\phi(f)) \widetilde{\Gamma}(W)-\pi(\phi(f)) \widetilde{\Gamma}(W)\right\|=\left|e^{-i \pi \lambda}-1\right|,
$$

in view of the CAR relations and the unitarity of $\widetilde{\Gamma}(W)$. Thus, we have a contradiction between (20) and (21), since the latter establishes that, for $\lambda \neq 0 \bmod 2$, the norm is a strictly positive constant. Finally, if $\lambda=0 \bmod 2$ the unitary implementers are local elements of $\mathscr{F}$. Indeed, if $W$ is centred in $\mathcal{O}$,

$$
\tilde{\Gamma}(W) \pi(\phi(f)) \tilde{\Gamma}(W)^{*}=\pi(\phi(f))
$$

for every $f$ with support spatially separated from $\mathcal{O}$. Therefore, by twisted duality, $\tilde{\Gamma}(W) \in \mathscr{F}\left(\mathcal{O}^{\prime}\right)^{\prime}=\mathscr{F}(\mathcal{O})^{\tau}$. On the other hand, since the twisting is involutive on any local field algebra, i.e. $\mathscr{F}(\mathcal{O})^{\tau \tau}=\mathscr{F}(\mathcal{O}), \tilde{\Gamma}(W) \in \mathscr{F}(\mathcal{O})$ follows from $\tilde{\Gamma}(W)^{\tau} \equiv \tilde{\Gamma}(W)$.

This result shows that the strange behaviour of statistics for this model appears only when the implementers are not elements of the field algebra, confirming that there is no contradiction between what we expected from the general theory of superselections sectors and the peculiarities arising from this model. When the "solitonic" parameter $\lambda$ vanishes, the statistics is again trivial, i.e. conventional Bose-Einstein or Fermi-Dirac. Since only the zero charge $\tilde{\Gamma}(W)$ are gauge invariant, the unique cases in which the implementers are observables (and local) are when $\lambda \in 2 \mathbb{Z}$ and $q=0$. The classification of the localizability property of our implementers is thus complete. 


\section{$5 \quad$ Braiding Structure and Asymptotic Abelianness}

In order to approach the study of strange statistics with a more general tool, we observe that the well known AQFT as formulated in [9, 10] and [12] cannot be applied here in its entirety, since Haag duality is violated by $(1+1)$ dimensional free massive Dirac field theory. A more appropriate setting seems to be that proposed in 2] in order to construct symmetric tensor $C^{*}$-categories in $\mathrm{QED}$, since it extends to theories where intertwiners are not contained in the algebra where the endomorphisms act. Moreover, the endomorphisms are not necessary locally inner ${ }^{3}$, but only in an asymptotic sense. Asymptotic abelianness yields a tensor $C^{*}$-category starting directly from representations, without exploiting Haag duality. We set up notation and state definitions.

Let us introduce two sets of nets $\rho_{W} \mapsto \mathcal{U}_{\rho_{W}}$ and $\rho_{W} \mapsto \mathcal{V}_{\rho_{W}}$ for every object $\rho_{W}$. Each net consists of unitary intertwiners in $\left(\rho_{W}, \rho_{W_{x_{m}}}\right)$, where $\rho_{W_{x_{m}}}$ tends pointwise in norm to the identity morphism on $\mathscr{A}$ for suitable sequences $\left\{x_{m}\right\}_{m}$.

Definition 2 (Asymptotic abelianness) A field theory model satisfies asymptotic abelianness if, given intertwiners $R \in\left(\rho_{W}, \rho_{W^{\prime}}\right), S \in\left(\rho_{\widetilde{W}}, \rho_{\widetilde{W}^{\prime}}\right)$ and nets $U_{m} \in \mathcal{U}_{\rho_{W}}, U_{m^{\prime}}^{\prime} \in \mathcal{U}_{\rho_{W^{\prime}}}, V_{n} \in \mathcal{V}_{\rho_{\widetilde{W}}}, V_{n^{\prime}}^{\prime} \in \mathcal{V}_{\rho_{\widetilde{W}^{\prime}}}$,

$$
U_{m^{\prime}}^{\prime} R U_{m}^{*} \times V_{n^{\prime}}^{\prime} S V_{n}^{*}-V_{n^{\prime}}^{\prime} S V_{n}^{*} \times U_{m^{\prime}}^{\prime} R U_{m}^{*} \longrightarrow 0
$$

in norm as $m, m^{\prime}, n, n^{\prime} \rightarrow \infty$.

Finally, the sets of nets must be compatible with products: for each pair $\rho_{W}, \rho_{W^{\prime}} \in \Delta$, there exist $U_{m} \in \mathcal{U}_{\rho_{W}}$ and $U_{m^{\prime}} \in \mathcal{U}_{\rho_{W^{\prime}}}$ such that $U_{m} \times U_{m^{\prime}} \in$ $\mathcal{U}_{\rho_{W} \rho_{W^{\prime}}}$, and similarly for $\mathcal{V}$. Here $\Delta$ denotes a semigroup of endomorphism of $\mathscr{A}$. If the nets satisfy all these conditions, they give rise to a bi-asymptopia.

Theorem 3 [2] If $\rho_{W}, \rho_{\widetilde{W}} \in \Delta$, then:

$$
\varepsilon\left(\rho_{W}, \rho_{\widetilde{W}}\right):=\lim _{m, n \rightarrow \infty} V_{n}^{*} \times U_{m}^{*} U_{m} \times V_{n}
$$

exists, is independent of $U_{m} \in \mathcal{U}_{\rho_{W}}$ and $V_{n} \in \mathcal{V}_{\rho_{\widetilde{W}}}$, and lies in $\left(\rho_{W} \rho_{\widetilde{W}}, \rho_{\widetilde{W}} \rho_{W}\right)$. Moreover, if $R \in\left(\rho_{W}, \rho_{W^{\prime}}\right), \quad S \in\left(\rho_{\widetilde{W}}, \rho_{\widetilde{W}^{\prime}}\right)$ and $\rho_{\widehat{W}} \in \Delta$, then:

$$
\begin{gathered}
\varepsilon\left(\rho_{W^{\prime}}, \rho_{\widetilde{W}^{\prime}}\right) \circ R \times S=S \times R \circ \varepsilon\left(\rho_{W}, \rho_{\widetilde{W}}\right), \\
\varepsilon\left(\rho_{W} \rho_{\widetilde{W}}, \rho_{\widehat{W}}\right)=\varepsilon\left(\rho_{W}, \rho_{\widehat{W}}\right) \times \mathbb{1}_{\rho_{\overparen{W}}} \circ \mathbb{1}_{\rho_{W}} \times \varepsilon\left(\rho_{\widetilde{W}}, \rho_{\widehat{W}}\right), \\
\varepsilon\left(\rho_{W}, \rho_{\widetilde{W}} \rho_{\widehat{W}}\right)=\mathbb{1}_{\rho_{\widetilde{W}}} \times \varepsilon\left(\rho_{W}, \rho_{\widehat{W}}\right) \circ \varepsilon\left(\rho_{W}, \rho_{\widetilde{W}}\right) \times \mathbb{1}_{\rho_{\widehat{W}}} .
\end{gathered}
$$

\footnotetext{
${ }^{3}$ We recall that $\widetilde{\Gamma}(W) \notin \mathscr{A}$ in almost all cases.
} 
We apply this method to our class $\Delta$ of automorphism in the setting of the $(1+1)$-dimensional free massive Dirac field. In light of the computation performed in the previous section, it remains to verify the condition of asymptotic abelianness in order to have a bi-asymptopia and consequently a braiding for our category. Starting from $(2+1)$-dimensional models, where a space-like cone $\mathcal{C}$ and its opposite $-\mathcal{C}$ are usually chosen as asymptotic localization regions in the definition of the families $\mathcal{U}$ and $\mathcal{V}$, we extends the method to a $(1+1)$-dimensional space-time by choosing the two standard wedges $W_{ \pm}$. Since in $(1+1)$-dimensions there is a natural notion of right and left, hence of $+\infty$ and $-\infty$, we set

$$
\begin{gathered}
\mathcal{U}_{\rho_{W}}:=\left\{\left(U_{a}\right)_{a} \subset\left(\rho_{W}, \rho_{W_{a}}\right), a \rightarrow+\infty\right\}, \\
\mathcal{V}_{\rho_{W}}:=\left\{\left(V_{b}\right)_{b} \subset\left(\rho_{W}, \rho_{W_{b}}\right), b \rightarrow-\infty\right\},
\end{gathered}
$$

where the two families of nets are contained in $W_{+}, W_{-}$respectively. (This condition implies that the nets are contained in the causal complement of every bounded region for large values of the indexes). Let $R: \rho_{W} \rightarrow \rho_{W^{\prime}}$, $S: \rho_{\widetilde{W}} \rightarrow \rho_{\widetilde{W}^{\prime}}$, and $z, \zeta \in \mathbb{C}$ be defined by

$$
R=z \widetilde{\Gamma}\left(W^{\prime} W^{*}\right), \quad S=\zeta \widetilde{\Gamma}\left(\widetilde{W^{\prime}} \widetilde{W}^{*}\right) .
$$

As intertwiners $U_{m} \in \mathcal{U}_{\rho_{W}}, U_{m^{\prime}}^{\prime} \in \mathcal{U}_{\rho_{W^{\prime}}}, V_{n} \in \mathcal{V}_{\rho_{\widetilde{W}}}, V_{n^{\prime}}^{\prime} \in \mathcal{V}_{\rho_{\widetilde{W}}}$, we set:

$$
\begin{aligned}
U_{m} & =\lambda_{m} \widetilde{\Gamma}\left(W_{x_{m}} W^{*}\right), & & x_{m} \stackrel{W_{+}}{\longrightarrow}+\infty \\
U_{m^{\prime}}^{\prime} & =\lambda_{m^{\prime}}^{\prime} \widetilde{\Gamma}\left(W_{x_{m^{\prime}}^{\prime}}^{\prime} W^{\prime *}\right), & & x_{m^{\prime}}^{\prime} \stackrel{W_{+}}{\longrightarrow}+\infty \\
V_{n} & =\mu_{n} \widetilde{\Gamma}\left(\widetilde{W}_{y_{n}} \widetilde{W}^{*}\right), & & y_{n} \stackrel{W_{-}}{\longrightarrow}-\infty \\
V_{n^{\prime}}^{\prime} & =\mu_{n^{\prime}}^{\prime} \widetilde{\Gamma}\left(\widetilde{W}_{y_{n^{\prime}}^{\prime}}^{\prime} \widetilde{W}^{\prime *}\right), & & y_{n^{\prime}}^{\prime} \stackrel{W_{-}}{\longrightarrow}-\infty
\end{aligned}
$$

where $\lambda_{m}, \lambda_{m^{\prime}}^{\prime}, \mu_{n}, \mu_{n^{\prime}}^{\prime} \in \mathbb{C}$ are defined as the scalar $z$, for every $m, m^{\prime}, n, n^{\prime}$. To simplify notation, in the sequel $x_{m}^{\prime}$ stands for $x_{m^{\prime}}^{\prime}$. The term of the sequence to which we refer will be clear from the context. Analogous simplifications will be adopted for $y_{n^{\prime}}^{\prime}, \lambda_{m^{\prime}}^{\prime} \mu_{n^{\prime}}^{\prime}$. We now perform the computation of the limit in (22), which now assumes the form:

$$
\begin{array}{rl} 
& \lambda_{m}^{\prime} \widetilde{\Gamma}\left(W_{x_{m}^{\prime}}^{\prime} W^{\prime *}\right) z \widetilde{\Gamma}\left(W^{\prime} W^{*}\right) \bar{\lambda}_{m} \widetilde{\Gamma}\left(W_{x_{m}} W^{*}\right)^{*} \times \\
& \times \mu_{n}^{\prime} \widetilde{\Gamma}\left(\widetilde{W}_{y_{n}^{\prime}}^{\prime} \widetilde{W}^{\prime *}\right) \zeta \widetilde{\Gamma}\left(\widetilde{W}^{\prime} \widetilde{W}^{*}\right) \bar{\mu}_{n} \widetilde{\Gamma}\left(\widetilde{W}_{y_{n}} \widetilde{W}^{*}\right)^{*}- \\
& -\mu_{n}^{\prime} \widetilde{\Gamma}\left(\widetilde{W}_{y_{n}^{\prime}}^{\prime} \widetilde{W}^{\prime *}\right) \zeta \widetilde{\Gamma}\left(\widetilde{W}^{\prime} \widetilde{W}^{*}\right) \bar{\mu}_{n} \widetilde{\Gamma}\left(\widetilde{W}_{y_{n}} \widetilde{W}^{*}\right)^{*} \times \\
\times & \lambda_{m}^{\prime} \widetilde{\Gamma}\left(W_{x_{m}^{\prime}}^{\prime} W^{\prime *}\right) z \widetilde{\Gamma}\left(W^{\prime} W^{*}\right) \bar{\lambda}_{m} \widetilde{\Gamma}\left(W_{x_{m}} W^{*}\right)^{*}= \\
=\lambda_{m}^{\prime} \bar{\lambda}_{m} z & D\left(W_{x_{m}^{\prime}}^{\prime} W^{\prime *}, W^{\prime} W^{*}\right) D\left(W_{x_{m}^{\prime}}^{\prime} W^{*}, W W_{x_{m}}^{*}\right) \widetilde{\Gamma}\left(W_{x_{m}^{\prime}}^{\prime} W_{x_{m}}^{*}\right) \times
\end{array}
$$




$$
\times \mu_{n}^{\prime} \bar{\mu}_{n} \zeta D\left(\widetilde{W}_{y_{n}^{\prime}}^{\prime} \widetilde{W}^{\prime *}, \widetilde{W}^{\prime} \widetilde{W}^{*}\right) D\left(\widetilde{W}_{y_{n}^{\prime}}^{\prime} \widetilde{W}^{*}, \widetilde{W} \widetilde{W}_{y_{n}}^{*}\right) \widetilde{\Gamma}\left(\widetilde{W_{y_{n}^{\prime}}^{\prime}} \widetilde{W}_{y_{n}}^{*}\right)-(\leftrightarrow),
$$

where $(\leftrightarrow)$ denotes the cross product of the same terms in the inverse order. Here $D(A, B)$ is the cocycle of the projective representation $\widetilde{\Gamma}$, i.e. $\widetilde{\Gamma}\left(W_{1}\right) \widetilde{\Gamma}\left(W_{2}\right)=D\left(W_{1}, W_{2}\right) \widetilde{\Gamma}\left(W_{1} W_{2}\right)$. Collecting all scalars in a factor $q$, (23) reduces to

$$
q\left(\widetilde{\Gamma}\left(W_{x_{m}^{\prime}}^{\prime} W_{x_{m}}^{*}\right) \times \widetilde{\Gamma}\left(\widetilde{W}_{y_{n}^{\prime}}^{\prime} \widetilde{W}_{y_{n}}^{*}\right)-\widetilde{\Gamma}\left(\widetilde{W}_{y_{n}^{\prime}}^{\prime} \widetilde{W}_{y_{n}}^{*}\right) \times \widetilde{\Gamma}\left(W_{x_{m}^{\prime}}^{\prime} W_{x_{m}}^{*}\right)\right)
$$

so, to evaluate the asymptotic behaviour of (22) it suffices to compute the limit of the expression in parentheses. Since

$$
\widetilde{\Gamma}\left(W_{x_{m}^{\prime}}^{\prime} W_{x_{m}}^{*}\right): \rho_{W_{x_{m}}} \longrightarrow \rho_{W_{x_{m}^{\prime}}^{\prime}}, \quad \widetilde{\Gamma}\left(\widetilde{W}_{y_{n}^{\prime}}^{\prime} \widetilde{W}_{y_{n}}^{*}\right): \rho_{\widetilde{W}_{y_{n}}} \longrightarrow \rho_{\widetilde{W}_{y_{n}^{\prime}}^{\prime}}
$$

the expression in (24) becomes:

$$
\begin{gathered}
q\left(\widetilde{\Gamma}\left(W_{x_{m}^{\prime}}^{\prime} W_{x_{m}}^{*}\right) \widetilde{\Gamma}\left(W_{x_{m}}\right) \widetilde{\Gamma}\left(\widetilde{W}_{y_{n}^{\prime}}^{\prime} \widetilde{W}_{y_{n}}^{*}\right) \widetilde{\Gamma}\left(W_{x_{m}}\right)^{*}-\right. \\
\left.-\widetilde{\Gamma}\left(\widetilde{W}_{y_{n}^{\prime}}^{\prime} \widetilde{W}_{y_{n}}^{*}\right) \widetilde{\Gamma}\left(\widetilde{W}_{y_{n}}\right) \widetilde{\Gamma}\left(W_{x_{m}^{\prime}}^{\prime} W_{x_{m}}^{*}\right) \widetilde{\Gamma}\left(\widetilde{W}_{y_{n}}\right)^{*}\right)= \\
=q^{\prime}\left(\widetilde{\Gamma}\left(W_{x_{m}^{\prime}}^{\prime}\right) \widetilde{\Gamma}\left(\widetilde{W}_{y_{n}^{\prime}}^{\prime}\right) \widetilde{\Gamma}\left(\widetilde{W}_{y_{n}}^{*}\right) \widetilde{\Gamma}\left(W_{x_{m}}\right)^{*}-\widetilde{\Gamma}\left(\widetilde{W}_{y_{n}^{\prime}}^{\prime}\right) \widetilde{\Gamma}\left(W_{x_{m}^{\prime}}^{\prime}\right) \widetilde{\Gamma}\left(W_{x_{m}}\right)^{*} \widetilde{\Gamma}\left(\widetilde{W}_{y_{n}}^{*}\right)\right)
\end{gathered}
$$

where

$$
q^{\prime}:=q \overline{D\left(W_{x_{m}^{\prime}}^{\prime}, W_{x_{m}}^{*}\right) D\left(\widetilde{W}_{y_{n}^{\prime}}^{\prime}, \widetilde{W}_{y_{n}}^{*}\right)} .
$$

By construction $y_{n}^{\prime}<x_{m}^{\prime}, y_{n}<x_{m}$ for $m, n$ sufficiently large, then unitaries $W_{x_{m}^{\prime}}^{\prime}$ and $\widetilde{W}_{y_{n}^{\prime}}^{\prime}$ are centred in disjoint intervals for $m, n$ sufficiently large. Equivalently, the corresponding implementers $\widetilde{\Gamma}\left(W_{x_{m}^{\prime}}^{\prime}\right)$ and $\widetilde{\Gamma}\left(\widetilde{W}_{y_{n}^{\prime}}\right)$ are localized in causally disjoint regions. We are then in a position to exploit the commutation rules between second quantization operators we have established in Sect. 2. For example,

$$
\widetilde{\Gamma}\left(W_{x_{m}^{\prime}}^{\prime}\right) \widetilde{\Gamma}\left(\widetilde{W}_{y_{n}^{\prime}}^{\prime}\right)=e^{-i \pi\left(n \lambda^{\prime}+n^{\prime} \lambda\right)} \widetilde{\Gamma}\left(\widetilde{W}_{y_{n}^{\prime}}^{\prime}\right) \widetilde{\Gamma}\left(W_{x_{m}^{\prime}}^{\prime}\right),
$$

and analogously for $W_{x_{m}}$. Performing the substitutions, it turns out that (22) vanishes for large values of the indexes and thus, a fortiori, tends to zero. We have thus shown the following

Proposition 9 The subcategory of $\operatorname{End}(\mathscr{A})$ generated from $\Delta$ admits a braiding structure $\varepsilon$. 
This model shows that the method of asymptotic abelianess, in the form stated in 2, cannot be applied to $(1+1)$-dimensional massive theories in order to obtain a generalized statistics operator, since the definition of biasymptopias is not consistent with the geometric peculiarity of space-time which may give rise to two distinct statistics operators. More precisely, we compare the braiding $\varepsilon$ of Theorem 3 with the statistics operator $\varepsilon\left(\rho_{W}, \rho_{W^{\prime}}\right)$ computed in the purely algebraic setting as in (14). If $\left\{x_{m}\right\}_{m}$ and $\left\{y_{n}\right\}_{n}$ are such that $\mathcal{O}_{x_{m}} \succ \mathcal{O}_{y_{n}}$ for sufficiently large $m$ and $n$, one has

$$
\begin{gathered}
V_{n}^{*} \times U_{m}^{*} U_{m} \times V_{n}= \\
=\bar{\mu}_{n} \widetilde{\Gamma}\left(\widetilde{W} \widetilde{W}_{y_{n}}^{*}\right) \times \bar{\lambda}_{m} \widetilde{\Gamma}\left(W W_{x_{m}}^{*}\right) \circ \lambda_{m} \widetilde{\Gamma}\left(W_{x_{m}} W^{*}\right) \times \mu_{n} \widetilde{\Gamma}\left(\widetilde{W}_{y_{n}} \widetilde{W}^{*}\right)= \\
\widetilde{\Gamma}\left(\widetilde{W} \widetilde{W}_{y_{n}}^{*}\right) \widetilde{\Gamma}\left(\widetilde{W}_{y_{n}}\right) \widetilde{\Gamma}\left(W W_{x_{m}}^{*}\right) \widetilde{\Gamma}\left(\widetilde{W}_{y_{n}}^{*}\right) \widetilde{\Gamma}\left(W_{x_{m}} W^{*}\right) \widetilde{\Gamma}(W) \widetilde{\Gamma}\left(\widetilde{W}_{y_{n}} \widetilde{W}^{*}\right) \widetilde{\Gamma}(W)^{*}
\end{gathered}
$$

which coincides with the expression of $\varepsilon\left(\rho_{W}, \rho_{\widetilde{W}}\right)$ gained by transporting $\rho_{W}$ and $\rho_{\widetilde{W}}$ resp. to $\rho_{W_{x_{m}}}$ and $\rho_{\widetilde{W}_{y_{n}}}$. Since the two nets of double cones are contained in distinct components of $\mathcal{O}^{\prime}$, this is incompatible with the basic prescriptions of AQFT, since this procedure would be equivalent to not remaining in a fixed connected component! We remark that asymptotic abelianness requires that the two nets of double cones $\mathcal{O}_{m}^{\mathcal{U}}$ and $\mathcal{O}_{n}^{\mathcal{V}}$, which appear in $\mathcal{U}_{\rho_{W}}$ and $\mathcal{V}_{\rho_{W}}$, do lie in distinct components of $\mathcal{O}^{\prime}$, since only this configuration guarantees that $\mathcal{O}_{m}^{\mathcal{U}}-\mathcal{O}_{n}^{\mathcal{V}}$ tends space-like to infinity as $m, n \rightarrow \infty$. (There are no alternatives in $(1+1)$-dimension with no additional constraint). So, the theory of bi-asymptopias may not lead to true statistics when the Minkowski space is not at least $(2+1)$-dimensional and braid statistics occurs. As in the AQFT setting, we can always collect path connected bi-asymptopias into equivalence classes, but without additional prescription on the double limit in Definition 2, we could include objects which have no physical meaning, since they correspond in (1+1)-dimensions to working with both components of $\mathcal{O}^{\prime}$ at the same time. A natural way to generalize this approach to bidimensional theories is to reformulate some definitions, giving a restricted notion of asymptotic abelianness appropriate to all dimensions. Instead of performing the double limit as in Definition 2 and Theorem 3, we choose a particular "direction", for example the diagonal one, i.e. $m=n$,

$$
\varepsilon\left(\rho_{W}, \rho_{\widetilde{W}}\right):=\lim _{n \rightarrow \infty} V_{n}^{*} \times U_{n}^{*} U_{n} \times V_{n},
$$

provided $\mathcal{O}_{x_{n}}$ and $\mathcal{O}_{y_{n}}$ are space-like separated for $m$ and $n$ sufficiently large. (Analogously for the definitions of asymptotic abelianness and biasymptopias). All properties continue to be valid, since the true reason for sending to infinity the two nets of double cones is to exploit morphisms which commute. For example, with the definition $\mathcal{O}_{n}^{\mathcal{V}}:=\mathcal{O}_{n}^{\mathcal{U}} \pm \hat{e}_{1} n$, all conditions are satisfied and each pair of nets lies in the wedge $W_{ \pm}$for large values of the 
indexes. (Here $\hat{e}_{1}$ denotes the unit vector in the $x_{1}$-direction). In this way, the braiding structure arising from asymptotic abelianness of intertwiners coincides with the braiding computed for a Haag-dual net whose morphisms are all inner, and it gives rise to a true braided tensor $C^{*}$-category in all other cases, e.g. the present one, (where the statistics operator now has a genuine, not simply formal, meaning of statistics). In other terms, we have excluded all cases in which intertwiners satisfy asymptotic abelianness, but the limits in Theorem 3 do not give rise to a statistics operator. The braiding induced by these bi-asymptopias is really non symmetric, since biasymptopias $\{\mathcal{U}, \mathcal{V}\},\{\mathcal{V}, \mathcal{U}\}$ are not path connected. In $(3+1)$ dimensions all particles exhibit ordinary statistics since we can choose $\mathcal{O}_{n}^{\mathcal{V}}=-\mathcal{O}_{n}^{\mathcal{U}}$ when we deal with strictly localized morphisms. This ensures the possibility of changing continuously from $\{\mathcal{U}, \mathcal{V}\}$ to $\{\mathcal{V}, \mathcal{U}\}$ along a chain of double cones. In $(2+1)$ dimensions, where cones give the better notion of localization, one can choose $\rho_{a}$ in such a way that $a$ tends to space-like infinity remaining in a space-like cone $\mathcal{C}$, resp. $-\mathcal{C}$, for $\mathcal{U}_{\rho}, \mathcal{V}_{\rho}$, and it is always possible to interchange the two cones by a sequences of allowed moves. This is not possible in $(1+1)$ dimensions, since $\mathcal{O}^{\prime}$ is not connected and thus, a fortiori, is not arcwise connected. We remark that a distinction between $\varepsilon(\rho, \sigma)$ and $\varepsilon(\sigma, \rho)^{*}$ for a generic pair of DHR morphisms cannot be achieved by interchanging the roles of $\mathcal{U}$ and $\mathcal{V}$, since both nets of double cones are in the same connected component of $\mathcal{O}^{\prime}$. Hence, in two dimensions to each morphism (object) we must associate two bi-asymptopias, one for each side of $\mathcal{O}^{\prime}$. A direct computation of the limits in Theorem 3 for each connected component separately, gives two distinct values, $e^{ \pm 2 \pi i n \lambda}$, which coincide with those found before.

Remark. Although two arbitrary automorphisms of the form $\rho_{W}$ are always connected by a similarity transformation (i.e., $\left.\rho_{W_{2}}=\operatorname{Ad} \widetilde{\Gamma}\left(W_{2} W_{1}^{*}\right) \circ \rho_{W_{1}}\right)$, this does not imply that they are unitarily equivalent through a local element of the observable algebra, since the unitary intertwiner is not necessarily in $\mathscr{A}$. More precisely, if $\left(n_{1}, \lambda_{1}\right) \neq\left(n_{2}, \lambda_{2}\right)$, then $\widetilde{\Gamma}\left(W_{1} W_{2}^{*}\right) \notin \mathscr{A}$ as a consequence of the previous observation and of group relations for unitaries $W(\cdot, \cdot ; \varepsilon)$, i.e. $W(n, \lambda) W\left(n^{\prime}, \lambda^{\prime}\right)=W\left(n+n^{\prime}, \lambda+\lambda^{\prime}\right), W(n, \lambda)^{*}=$ $W(-n,-\lambda)$. On the other hand, products $W_{x} W^{*}$ have a different behaviour, since

$$
W(n, \lambda ; \varepsilon)_{x} W(n, \lambda ; \varepsilon)^{*}=W\left(n, \lambda ; \tau_{x} \varepsilon-\varepsilon\right)
$$

and, as already stated, $\widetilde{\Gamma}\left(W_{x} W^{*}\right) \in \mathscr{A}(\tilde{\mathcal{O}})$, where $\tilde{\mathcal{O}} \supset \mathcal{O} \cup \mathcal{O}_{x}$. We emphasize that the notation in (25) may give rise to ambiguities, since the operator on the right hand side carries no charge even if $n \neq 0$, due to the particular form of the generating function. 


\section{Conclusions}

In the setting of AQFT we have shown that a family of localized and transportable automorphisms of the observable algebra $\mathscr{A}$ exhibits non ordinary statistics. Inside each sector one has different braiding structures labelled by a solitonic parameter $\lambda$ which reflects the action of smeared-out kink operators carrying no charge.

Owing to non locality of charge implementers, statistics is not an invariant of the sector, as already known in some two-dimensional particle theories or in solitonic theories. On the underlying ordinary structure, smeared-out kink operators give rise to a continuous family of braided tensor categories in the sense of the theory of bi-asymptopias.

The results are consistent with AQFT, which must be handled carefully here when tackling problems arising from the non locality of unitary implementers, the violation of Haag duality and the topological peculiarity of $(1+1)$-dimensional space-time. Owing to the latter, some results of local field theory are no longer valid in a two-dimensional world, giving rise to a range of intermediate situations and strengthening the concept that for massive theories in $(1+1)$ dimensions statistics is not an intrinsic characteristic of sectors a priori 23]. In the present case, since Haag duality can be overcome by peculiarities of the model, strangeness of statistics has its origin in the fact that implementers do not lie in the field algebra.

The interpretation of the braiding structure of this model extends to the CAR algebra the constructive method exploited for the Weyl algebra. Since not all the braidings obtained in this way give rise to a notion of statistics compatible with the DHR analysis, but only those constructed from pairs of sets of nets which tend to the same space-like infinity, the method of biasymptopias can be carried over to $(1+1)$-dimensional space-time only if we add a compatibility condition. This kind of selection criterion reflects the "initial condition" which determines uniquely the statistics operator in the standard algebraic approach, i.e. trivialization of $\varepsilon\left(\rho_{W}, \rho_{W^{\prime}}\right)$ for $\rho_{W^{\prime}} \prec \rho_{W}$ [13. The particles described by this model are "statistical schizons", since the same sector allows "pseudo" statistical descriptions and they exist in the same Hilbert space either as bosons or as fermions or as proper anyons [22, i.e. in two-dimensional massive theories not only the spin but also the statistics is a convention.

\section{Acknowledgement}

I am greatly indebted to the supervisor of my $\mathrm{PhD}$ thesis, S. Doplicher, for many helpful discussions and much encouragement. I would like to thank C. D'Antoni, J. Roberts and M. Gabriel for a careful reading of the manuscript, and B. Schroer for useful correspondence. It is a pleasure to thank A. Silva, 
who was the coordinator of the $\mathrm{PhD}$ program at the Department of Mathematics of "La Sapienza", University of Rome, in the period when this work has been carried out.

\section{References}

[1] C. Adler. Braid group statistics in two-dimensional quantum field theory. Rev. Math. Phys. 7, 907-924 (1996)

[2] D. Buchholz, S. Doplicher, G. Morchio, J. E. Roberts, F. Strocchi. Asymptotic abelianness and braided tensor $C^{*}$-categories. arXiv:math-ph/0209038

[3] D. Buchholz, G. Lechner. Modular nuclearity and localization. Annales Henri Poincare 5, 1065-1080 (2004)

[4] H. Baumgärtel, M. Jurke, F. Lledó. Twisted duality of the CAR-algebra. J. Math. Phys. 43, 4158-4179 (2002)

[5] A. L. Carey, S. N. M. Ruijsenaars. On fermionic gauge groups, current algebras and Kac-Moody algebras. Acta Appl. Math. 10, 1-86 (1987)

[6] A. L. Carey, C. A. Hurst, D. M. O'Brien. Automorphisms of the canonical anticommutation relation and index theory. J. Funct. Anal. 48, 360-393 (1982)

[7] S. Doplicher, R. Haag, J. E. Roberts. Fields, observables and gauge transformations I. Commun. Math. Phys. 1, 1-23 (1969)

[8] S. Doplicher, R. Haag, J. E. Roberts. Fields, observables and gauge transformations II. Commun. Math. Phys. 15, 173-200 (1969)

[9] S. Doplicher, R. Haag, J. E. Roberts. Local observables and particle statistics I. Commun. Math. Phys. 23, 199-230 (1971)

[10] S. Doplicher, R. Haag, J. E. Roberts. Local observables and particles statistics II. Commun. Math. Phys. 35, 49-85 (1974)

[11] S. Doplicher, J. E. Roberts. $C^{*}$-algebras an duality for compact groups: why there is a compact group of internal symmetries in particle physics. Proceedings of the International Conference on Mathematical Physics, Marseille (1986)

[12] S. Doplicher, J. E. Roberts. Why there is a field algebra with a compact gauge group describing the superselection structure in particle physics. Commun. Math. Phys. 131, 51-107 (1990) 
[13] K. Fredenhagen, K.-H. Rehren, B. Schroer. Superselection sectors with braid group statistics and exchange algebras II: geometric aspects and conformal covariance. Reviews in Mathematical Physics, Special Issue, 113-157 (1992)

[14] R. Köberle, E. C. Marino, Duality, mass spectrum and vacuum expectation values. Phys. Lett. B126, 475-480 (1983)

[15] M. Mueger. Superselection structure of massive quantum field theories in $1+1$ dimensions. Rev. Math. Phys. 10, 1147-1170 (1998)

[16] M. Mueger. Quantum double actions on operator algebras and orbifold quantum field theories. Commun. Math. Phys. 181, 137-181 (1998)

[17] J. Mund. No-go theorem for 'free' relativistic anyons in $d=2+1$. Lett. Math. Phys. 43, 319-328 (1998)

[18] A. Pressley, G. Segal. Loop groups. Clarendon Press (1986)

[19] J. E. Roberts. Lectures on algebraic quantum field theory. The algebraic theory of superselection sectors: Introduction and recent results. Singapore, World Scientific (1990)

[20] S. N. M. Ruijsenaars. The Wightman axioms for the fermionic Federbush model. Commun. Math. Phys. 87, 181-228 (1982)

[21] S. N. M. Ruijsenaars. Index formulas for generalized Wiener-Hopf operators and boson-fermion correspondence in $2 N$ dimensions. Commun. Math. Phys. 124, 553-593 (1989)

[22] B. Schroer. Scattering properties of anyons and plektons. Nucl. Phys. B369, 478-498 (1992)

[23] B. Schroer. Two-dimensional models as testing ground for principles and logarithmic structures. Annals of Physics 321, 435-479 (2006)

[24] B. Schroer, J. A. Swieca. Spin and statistics of quantum kinks. Nucl. Phys. B121, 505-513 (1977)

[25] F. Wilczek. Quantum mechanics of fractional spin particles. Phys. Rev. Lett. 49, 957-1149 (1983) 